\title{
WAVELET TRANSFORMS IN GENERALIZED FOCK SPACES
}

\author{
JOHN SCHMEELK \\ Department of Mathematical Sciences \\ Virginia Commonwealth University \\ Richmond, Virginia 23284-2014, U.S.A. \\ and \\ ARPAD TAKACI \\ Institute of Mathematics \\ University of Novi Sad \\ TRG D. OBRADOVICA 4, 21000 Novi Sad \\ Yugoslavia
}

(Received March 26, 1996 and in revised form May 27, 1996)

\begin{abstract}
A generalized Fock space is introduced as it was developed by Schmeelk [1-5], also Schmeelk and Takaci [6-8]. The wavelet transform is then extended to this generalized Fock space. Since each component of a generalized Fock functional is a generalized function, the wavelet transform acts upon the individual entry much the same as was developed by Mikusinski and Mott [9] based upon earlier work of Mikusinski and Taylor [10]. It is then shown that the generalized wavelet transform applied to a member of our generalized Fock space produces a more appropriate functional for certain applications.
\end{abstract}

KEY WORKS AND PHRASES: Generalized Fock functional, wavelets, bounded intersection property, generalized wavelet transforms.

1991 AMS SUBJECT CLASSIFICATION CODES: 46F12, 46F99

\section{INTRODUCTION}

Wavelet transform techniques are rapidly becoming a primary mathematical theory being implemented in many applications. The current development by the Federal Bureau of Investigation (FBI) in establishing a proper wavelet transform to store its $\mathbf{3 0}$ million criminal fingerprints now stored in filing cabinets illustrates the wavelet applicational importance. The advantage will not only be to compress the data files but to develop a faster and superior method to facilitate the matching process. These techniques are developed and discussed in a work of Strange [11].

Wavelets are also shown to be of significant importance in the music industry. A linear combination of three wavelets are shown to be an excellent model for the sound of the crash of cymbals in an orchestra shown by Von Baeyer [12]. The application of wavelets to seismic data developed by Grossmann and Morlet in reference [13] also illustrates the marvelous analysis of what the authors of this paper will term, "rough data". Perhaps in some situations the removal of the "overshoot" known as the Gibbs phenomenon in Fourier analysis gives wavelet analysis a key advantage in approximating our so called "rough" data discussed by Walter [14]. 
A natural question to ask is how can we apply wavelets to distributions? In particular how to apply them to singular distributions or non-regular distributions such as the Dirac delta functional which again in the authors opinion is a very "rough" object. Several research papers regarding the implementation of wavelets to distributions can be found in the research done by Walter [14-20] and Mikusinski and Mott [9]. We will extend several of these results.

The application of wavelets to image processing has been developed as a convolution operator in the work of Mallat [21-24] and further developed in a mathematical milieu in the work of Mikusinski and Mott [9].

We will first give a general overview of wavelet transforms considered as convolution operators for real valued functions belonging to the space, $L^{2}\left(g_{B}^{q}\right), q \geq 2$. We will not repeat here the marvelous multiresolution analysis conducted in the space, $L^{2}\left(\mathscr{R}^{2}\right)$, by Mallat [2124] and Meyer [25] and generalized to the space, $L^{2}\left(\mathscr{P}^{q}\right), q>2$ in the work of Daubechies [26]. We are also interested in the space $L^{2}\left(\Re^{2}\right)$ since an immediate application can then be made to image processing. The real valued functions in this application take on integer values ranging from 0 to 255 depending on the shade of gray contained in a black and white image and its argument is a member of $\mathscr{R}^{2}$ designating the location within the image developed in Lim [27].

Moreover many signals in signal processing are bounded real valued functions on $T_{0}^{q}$ and are also members of the space, $L^{1}\left(\Re^{q}\right)$. Thus we are naturally lead to study the space, $L_{B}^{1}\left(\mathscr{T}_{B}^{q}\right)$, namely the space of bounded functions which are also members of $L^{2}\left(\mathscr{B}^{q}\right)$. Moreover it is well known that the convolution of two functions belonging to $L^{1}\left(\mathscr{B}^{q}\right)$ exists almost everywhere.

We then move on and consider the wavelet transform applied to classical distributional spaces. This then culminates by applying generalized wavelet transforms to our so-called generalized Fock spaces. The generalized Fock spaces are developed by Schmeelk [1-5] and Takaci [6-8]. A rapid review of the key features for these spaces are included in section 3. The three representations of our generalized Fock functionals will enable us to consider a form of classical Fock spaces as well as generalized Fock spaces. These so-called generalized Fock functionals contain functionals oftentimes non-linear which may consist of an infinite array of translated Dirac delta functionals. This statement will become clear as the paper develops the surrounding mathematical considerations. It is the wavelet transform generalizations to these spaces which will provide us with the authors so-called "somewhat smooth" or "very smooth" representations for the "very rough" functionals.

\section{SOME NOTIONS AND NOTATIONS}

We recall some properties for our sequences of real numbers, $\mu=\left(m_{q}\right)_{q \in N_{0}}$, and sequences of functions, $\mathcal{H}=\left(M_{p}(\cdot)\right)_{p \in N_{0}}$. The sequences of real numbers, $\mu=\left(m_{q}\right)_{q \epsilon N_{0}}$, satisfy the Komatsu conditions [28], namely

$$
m_{q}^{2} \leq m_{q-1} \cdot m_{q+1}, q \in N
$$

(M.2) there exist real numbers, $A$ and $H$, such that $m_{q+1} \leq A \cdot H^{q} \cdot m_{q}, q \in N_{0}$;

(M.3) $\quad \sum_{q=1}^{\infty} \frac{m_{q}-1}{m_{q}}<\infty$. 
These conditions are commonly called logarithmic convexity, stability under ultra differential operators and strong non-quasi analyticity respectively. It is convenient to select $m_{0}=1$. One easily verifies, for instance, the sequence,

satisfies the three conditions.

$$
m_{q}=q^{q s}, s>1
$$

We next select a sequence of continuous functions, $\mathcal{H}=\left(M_{p}(\cdot)\right)_{p \in N_{0}}$, on $\Re^{q}, q \geq 1$. We require the traditional conditions. $(P),(M)$ and $(N)$ hold (see Gelfand and Shilov [29] or Pilipovic and Takači [30] ) as well as the inequalities,

$$
1 \triangleq M_{0}\left(t_{1}, \ldots, t_{q}\right) \leq \cdots \leq M_{p}\left(t_{1}, \ldots, t_{q}\right) \leq \cdots .
$$

Then an infinitely differentiable function, $\phi_{q}\left(t_{1}, \ldots, t_{q}\right)$, is in the test space, $96(\mathcal{H})$, if and only if for each $p \in N_{0}$ the following norms are finite;

$$
\begin{gathered}
\left\|\phi_{q}\right\|_{p}=\sup \left\{\left|\phi^{\left(j_{1}, \ldots, j_{q}\right)}\right|\left(t_{1}, \ldots, t_{q}\right) \mid M_{p}\left(t_{1}, \ldots, t_{q}\right):\right. \\
\left.t \in \Re^{q}, \quad j_{i} \leq p, \quad 1 \leq i \leq q\right\}
\end{gathered}
$$

where

$$
\phi^{\left(j_{1}, \ldots, j_{q}\right)}\left(t_{1}, \ldots, t_{q}\right)=\frac{\partial^{j_{1}+\cdots+j_{q}}}{\partial^{j_{1}} t_{1} \ldots \partial^{j_{q}} t_{q}} \phi\left(t_{1}, \ldots, t_{q}\right)
$$

and $t=\left(t_{1}, \ldots, t_{q}\right) \in \Re^{q}$.

The family of norms, $\left(\|\cdot\|_{p}\right)_{p \in N_{0}}$, defines a locally convex topology on the vector space, 96(值) which in view of condition $(P)$ turns it into a Fréchet space. The classical test functions, $\mathscr{T}\left(\Re^{q}\right)$, consisting of infinitely differentiable functions having compact support equipped with its usual topology is then dense in $96(\mathcal{M})$. This implies that the space of linear continuous functionals referred to as generalized functions on the test space, $96(\mathcal{M})$, and denoted $96^{\prime}(\mathcal{A})$ is in fact a countably normed space equipped with norms,

$$
\|x\|_{-p}=\sup \left\{|\langle x, \phi\rangle|:\|\phi\|_{p} \leq 1, \phi \in \mathscr{G}(\mathcal{H}), \quad p, \epsilon N_{0}\right\} .
$$

Moreover we observe that

$$
\|x\|_{0} \geq\|x\|_{-1} \geq \cdots \geq\|x\|_{-p} \geq \cdots
$$

for any $x \in \mathscr{G}^{\prime}(\mathcal{M})$. During our sequel we will occasionally use the functions, $\mathscr{H}=\left(M_{p}\right)_{p \in N_{0}}$, to be defined as

$$
M_{p}\left(t_{1}, \ldots, t_{q}\right)=\left[\left(1+t_{1}^{2}\right) \ldots\left(1+t_{q}^{2}\right)\right]^{p}
$$

whereby the test space, $\mathscr{S}(\mathcal{M})$ then becomes the test space of rapid descent, $\varphi^{(}\left(\Re^{q}\right)$ and $9^{\prime}(\mathfrak{M})$ becomes the Schwartz space of tempered distributions, $\mathscr{\Psi}^{\prime}\left(\Re^{q}\right)$.

\section{GENERALIZED FOCK SPACES}

Let $x \in 96^{\prime}(\mathcal{H})$ and consider 


$$
a_{q}: 96^{\prime}(\mathcal{M}) \times \cdots \times 96^{\prime}(\mathcal{M}) \mapsto \mathrm{C}
$$

a multilinear continuous functional on $q$-copies of $96^{\prime}(\mathcal{H})$ to $\mathrm{C}$. By definition we take $a_{0} \in \mathrm{C}$. We define

$$
\left\|a_{q}\right\|_{p}=\sup \left\{\left|a_{q}[x, \ldots, x]\right|: x \in 9 G^{\prime}(\mathcal{M}),\|x\|_{-p} \leq p \in \mathbb{N}\right\} .
$$

Then the infinite column vector,

$$
\Phi \leftrightarrow\left[\begin{array}{c}
a_{0} \\
a_{1} \\
\vdots \\
a_{q} \\
\vdots
\end{array}\right]
$$

is in the space, $\Gamma^{s, \mu, \mathcal{H}}, s>1$, if and only if the norm

$$
\|\| \Phi||_{s, \mu, M b}^{(p)}=\sup _{q \in N_{0}} \frac{\left\|a_{q}\right\|_{p} \cdot m_{q}}{s^{q}}
$$

is finite for every $p \in \mathbb{N}_{0}$.

Clearly the canonical inclusion,

$$
\Gamma^{s, \mu, \mathcal{H}} \mapsto \Gamma^{s^{\prime}, \mu, \mathcal{M}}
$$

is continuous provided $s^{\prime}>s>1$.

DEFINITION 3.6. A generalized Fock space, $\Gamma^{\mu, \mathcal{A}}$, is the inductive limit of the spaces, $\Gamma^{s, \mu, \mathcal{A}}$, which is denoted as

$$
\Gamma^{\mu, \mathcal{A b}}=\operatorname{ind}_{s \rightarrow \infty} \Gamma^{s, \mu, \mathcal{H}} .
$$

It was shown by Schmeelk [2] that each functional, $\Phi \leftrightarrow \Gamma^{\mu, \mathcal{A l}}$, has a kernel representation. This representation was shown to be

$$
\Phi \leftrightarrow\left[\begin{array}{c}
\phi_{0} \\
\phi_{1}\left(t_{1}\right) \\
\vdots \\
\phi_{q}\left(t_{1}, \ldots, t_{q}\right) \\
\vdots
\end{array}\right]
$$

where $\phi_{0}=a_{0} \in \mathrm{C}$ and $\phi_{q}\left(t_{1}, \ldots, t_{q}\right), q \geq 1$ are symmetric rapid descent test functions whenever the functions, $\mathcal{H}=\left(\mathcal{H}_{p}(\cdot)\right)_{p \in N_{0}}$ are defined as in expression (2.4). Moreover our functional representation given in expression (3.7) will then satisfy the norm conditions,

$$
\|\mid \Phi\|_{s, \mu, \mathcal{M}}^{(p)}=\sup _{q \in N_{0}} \frac{\left\|\phi_{q}\right\|_{p} \cdot m_{q}}{s^{q}}<\infty
$$

for every $p \in \mathbb{N}_{0}$ where $\left\|\phi_{q}\right\|_{p}$ is the norm defined in expression (2.2). The representations 
(3.3) or (3.7) enjoy a generalized square summable property as seen in the following theorem oftentimes postulated for classical Fock functionals.

THEOREM 3.9. Given a $\Phi \in \Gamma^{s, \mu, \mathcal{M}}, s>1$ then its kernel representation given in expression (3.7) satisfies

$$
\|\Phi\| \triangleq\left|\phi_{0}\right|^{2}+\sum_{q=1}^{\infty} \int_{\Re^{q}}\left|\phi_{q}\left(t_{1}, \ldots, t_{q}\right)\right|^{2} d t_{1} \ldots, d t_{q}<\infty .
$$

PROOF: See Schmeelk [6], Theorem 3.1.

The dual space, $\left(\Gamma^{\mu, \mathcal{M}}\right)^{\prime}$, was developed in reference [38-40] and each member, $F \in\left(\Gamma^{\mu, \mathcal{A}}\right)^{\prime}$, was shown to have the representation,

$$
F \leftrightarrow\left[F_{0}, F_{1}, \ldots, F_{q}, \ldots\right],
$$

where $F_{0} \in C$ and each $F_{q}, q \geq 1$ belongs to the generalized function space, $\mathcal{G}^{\prime}(\mathcal{A})$, and they all have an order $\leq p$. Moreover it was shown that each $F$ satisfies the condition,

$$
\sum_{q=0}^{\infty}\left\|F_{q}\right\|-p \frac{s^{q}}{m_{q}}\|\Phi\|_{s, \mu, \mu}^{(p)}<\infty
$$

for some fixed order, $p$.

The duality between $\Phi \in \Gamma^{\mu, \mathcal{H}}$ and $F \in\left(\Gamma^{\mu, \mathcal{H}}\right)^{\prime}$ is then defined by

$$
\left\langle\left\langle F_{1}, \Phi\right\rangle\right\rangle=\sum_{q=0}^{\infty}\left\langle F_{q}, \phi_{q}\right\rangle_{q}
$$

The notation in expression (3.12) given as $\left\langle F_{q}, \phi_{q}\right\rangle_{q}$ denotes the duality between the test space, $96(\mathcal{A b})$, and its corresponding generalized function space, $96^{\prime}(\mathcal{A b})$.

We now observe that our dual space, $\left(\Gamma^{\mu, \mathcal{H}}\right)^{\prime}$, can be equipped with the norms,

$$
\|F\|_{s, \mu, \Lambda b}^{(-p)} \triangleq \sum_{q=0}^{\infty}\left\|F_{q}\right\|_{-p} \frac{s^{q}}{m_{q}}
$$

where $p \geq$ order of all $\left\{F_{q}\right\}_{q=1}^{\infty}$ in the representation given in expression (3.10). Moreover whenever the series in (3.13) converges, our generalized Fock functional is a member of $\left(\Gamma^{\mu, 16}\right)^{\prime}$.

\section{WAVELET TRANSFORMS}

For this section and hereafter we consider the following space inclusions,

$$
\mathscr{T}\left(\Re^{q}\right) \subset \mathscr{Y}\left(\Re^{q}\right) \subset L_{B}^{1}\left(\Re^{q}\right) \subset L^{2}\left(\Re^{q}\right) \subset \mathscr{Y}^{\prime}\left(\Re^{q}\right) \subset \mathscr{D}^{\prime}\left(\Re^{q}\right) .
$$

Here again $L_{B}^{1}\left(\Re^{q}\right)$ is the space of bounded functions on $\Re^{q}, q \geq 1$ which are also members of $L^{2}\left(\Re^{q}\right)$. The tempered distributions, $\varphi^{\prime}\left(\Re^{q}\right)$ and distributions, $\mathscr{I}^{\prime}\left(\Re^{q}\right)$ are acting on rapid descent and compact support test functions respectively having independent variables belonging to $\Re^{q}$. Moreover we want to preserve the mathematical analysis surrounding the Fourier analysis developed in $\varphi^{\prime}\left(\Re^{q}\right)$. A principle result oftentimes termed the exchange principle is that the Fourier transform of a convolution is the product of the Fourier 
transforms. This is very important in applications since many software packages contain algorithms for Fast Fourier transforms, but do not support wavelet transform techniques. After we establish the wavelet transform to be a form of convolution, then these software packages via the exchange principle can be extended to do wavelet transforms.

The wavelet analysis surrounding this section to include the mother wavelet, the admissibility condition and multiresolution analysis can be found in Akansu [31], Benedetto and Frazier [32], Beylkin et al [33], Holschneider [34], Kaiser [35], Koornwinder [36], Ruskai [37] and Schumaker and Webb [38]. Also the inversion of the wavelet transform stemming from the admissibility condition can also be found within these references. We will begin our analysis by considering the wavelet transform as a special type of convolution as in Mallat [21-24] and Milusinski and Mott [9]. To this end we will adopt the following notation conventions.

If a function, $\phi(t) \in L^{2}\left(\Re_{q}^{q}\right)$, together with two $q$-tuples of real numbers, $a=\left(a_{i}\right)_{i=1}^{q}$, $a_{i} \neq 0,(1 \leq i \leq q)$ and $\tau=\left(\tau_{i}\right)_{i=1}^{q}$ are given, then the dilation and translation of $\phi(t)$ is

$$
\phi^{a, \tau}(t)=\frac{1}{\sqrt{\prod_{i=1}^{q} a_{i}}} \phi\left(\frac{t_{1}-\tau_{1}}{a_{1}}, \ldots, \frac{t_{q}-\tau_{q}}{a_{q}}\right) .
$$

Moreover we denote by $\phi_{a}(t)$ the function defined as

$$
\phi_{a}(t)=\frac{1}{\sqrt{\prod_{i=1}^{q} a_{i}}} \phi\left(\frac{t_{1}}{-a_{1}}, \ldots, \frac{t_{q}}{-a_{q}}\right) .
$$

Throughout our sequel we will always require the dilation sequence, $a=\left(a_{i}\right)_{i=1}^{q}$ satisfy the requirements, $a_{i} \neq 0, \quad(1 \leq i \leq q)$.

LEMMA 4.4 Given a function, $\phi(t) \in L^{2}\left(\Re^{q}\right)$

then $\|\phi(t)\|_{L^{2}\left(\Re^{q}\right)}=\left\|\phi^{a, \tau}(t)\right\|_{L^{2}\left(\Re^{q}\right)}=\left\|\phi_{a}(t)\right\|_{L^{2}\left(\Re^{q}\right)}$, where $\|\cdot\|_{L^{2}\left(\Re^{q}\right)}$

is the standard norm on the equivalence classes contained in $L^{2}\left(\Re^{q}\right)$.

PROOF. Follows directly from the change of variables, $T_{i}=\frac{t_{i},-\tau_{i}}{a_{i}}$ and $T_{i}=\frac{t_{i}}{a_{i}}$, $(1 \leq i \leq q)$ respectively. This lemma shows us that the energy in a real valued signal remains unchanged under the constructions, $\phi^{a, \tau}(t)$ and $\phi_{a}(t)$.

We now select a mother wavelet, $\psi(t) \in L_{B}^{1}\left(\Re^{q}\right)$, and define the wavelet transform of a function, $f(t) \in L^{1}\left(\Re^{q}\right)$, to be

$$
\begin{aligned}
& W_{f}(a, \tau) \triangleq \int_{-\infty}^{\infty} \cdots \int_{-\infty}^{\infty} f(t) \psi^{a, \tau}(t) d t \\
& =\int_{-\infty}^{\infty} \ldots \int_{-\infty}^{\infty} f\left(t_{1}, \ldots, t_{q}\right) \frac{1}{\sqrt{\prod_{i=1}^{q} a_{i}}} \psi\left(\frac{t_{1}-\tau_{1}}{a_{1}}, \ldots, \frac{t_{q}-\tau_{q}}{a_{q}}\right) d t_{1} \ldots d t_{q} \\
& =\int_{-\infty}^{\infty} \ldots \int_{-\infty}^{\infty} f\left(t_{1}, \ldots, t_{q}\right) \frac{1}{\sqrt{\prod_{i=1}^{q} a_{i}}} \psi\left(\frac{\tau_{1}-t_{1}}{-a_{1}}, \ldots, \frac{\tau_{q}-t_{q}}{-a_{q}}\right) d t_{1} \ldots d t_{q}
\end{aligned}
$$




$$
=\left[f(t) * \psi_{a}(t)\right](\tau),
$$

where $*$ denotes the convolution product of the functions, $f(t)$ and $\psi_{a}(t)$ as defined by Zemanian [39].

As an example of a mother wavelet we consider a form of the Meyer wavelet presented by Saitoh [40],

$$
\psi\left(t_{1}, \ldots, t_{q}\right)=\left[\frac{2 \pi^{-1 / 4}}{\sqrt{3}}\right]^{q}\left(1-t_{1}^{2}\right) \ldots\left(1-t_{q}^{2}\right) e^{\frac{-t_{1}^{2}-\ldots-t_{q}^{2}}{2}} .
$$

which satisfies the admissibility condition,

$$
\int_{-\infty}^{\infty} \ldots \int_{-\infty}^{\infty} \psi\left(t_{1}, \ldots, t_{q}\right) d t_{1} \ldots d t_{q}=0
$$

and moreover

$$
\int_{-\infty}^{\infty} \ldots \int_{-\infty}^{\infty}\left|\psi\left(t_{1}, \ldots, t_{q}\right)\right|^{2} d t_{1} \ldots d t_{q}=1
$$

The mother wavelet given in expression (4.6) is a rapid descent test function giving us a clear connection between wavelet analysis and tempered distributions. By applying the Schwartz inequality in the space, $L^{2}\left(\Re^{q}\right)$, we immediately have for our mother wavelet $(4.6)$ and any $f(t) \in L^{2}\left(\Re^{q}\right)$ the following inequality.

$$
\begin{aligned}
& \left|W_{f}(a, \tau)\right|=\left|\left(f(t), \psi^{a, \tau}(t)\right)_{L^{2}\left(\Re^{q}\right)}\right| \\
& \quad \leq\|f(t)\|_{L^{2}\left(\Re^{q}\right)} \cdot\left\|\psi^{a, \tau}(t)\right\|_{L^{2}\left(\Re^{q}\right)} \\
& \quad=\|f(t)\|_{L^{2}\left(\Re^{q}\right)} .
\end{aligned}
$$

This inequality will remain valid for any mother wavelet, $\psi^{a, \tau}(t)$, which has been normalized in the $L^{2}\left(\Re^{q}\right)$ norm.

\section{WAVELET TRANSFORMS OF GENERALIZED FUNCTIONS}

We briefly recall the definition of the convolution product for two generalized functions developed by Vladimirov [41] and Zemanian [39].

DEFINITION 5.1. Given two generalized functions, $F, G$, then their convolution product is

$$
\begin{aligned}
& \langle F * G, \phi\rangle=\left\langle F\left(t_{1}, \ldots, t_{q}\right),\left\langle G\left(\tau_{1}, \ldots, \tau_{q}\right), \phi\left(t_{1}+\tau_{1}, \ldots, t_{q}+\tau_{q}\right)\right\rangle\right\rangle= \\
& \int_{-\infty}^{\infty} \ldots \int_{-\infty}^{\infty} F\left(t_{1}, \ldots, t_{q}\right) G\left(\tau_{1}, \ldots, \tau_{q}\right) \phi\left(t_{1}+\tau_{1}, \ldots, t_{q}+\tau_{q}\right) d t_{1}, \ldots, d t_{q} d \tau_{1}, \ldots, d \tau_{q}
\end{aligned}
$$

provided the above equality (5.2) is well defined for all test functions, $\phi(t) \in \mathscr{T}\left(\Re^{q}\right)$. The integral representation given in (5.2) is valid only for regular generalized functions. 
Inherent in definition 5.1 is the problem with the construction $\phi(t+\tau)$ which destroys the compact support and polynomial growth condition when the test function, $\phi(t)$, belongs to $\mathscr{D}\left(\Re^{q}\right)$ or $\mathscr{Y}\left(\Re^{q}\right)$ respectively. To overcome this difficulty it is shown by Vladimirov [41] and Zemanian [39] that if $F$ and $G$ both have compact support, or both have bounded supports on the left or the right, then the convolution product is well defined. Moreover it is shown by Vladimirov [41] that the convolution product remains well defined for $F \in \Psi^{\prime}\left(\Re^{q}\right)$ and $G$ a generalized function having compact support.

Since the convolution of the translated generalized derivative of the Dirac functional, $\delta^{\left(j_{1}, \ldots, j_{q}\right)}\left(\tau_{1}-\tau_{1}^{0}, \ldots, \tau_{q}-\tau_{q}^{0}\right)$, and a distribution, $F$, is important to our sequel, we compute it

implementing the notation convention, $|j|=\sum_{i=1}^{q} j_{i}$.
This gives us

$$
\begin{aligned}
& \left\langle F * \delta^{\left(j_{1}, \ldots, j_{q}\right)}\left(\tau_{1}-\tau_{1}^{0}, \ldots, \tau_{q}-\tau_{q}^{0}\right), \phi\right\rangle= \\
& \left\langle F\left(t_{1}, \ldots, t_{q}\right),\left\langle\delta^{\left(j_{1}, \ldots, j_{q}\right)}\left(\tau_{1}-\tau_{1}^{0}, \ldots, \tau_{q}-\tau_{q}^{0}\right), \phi\left(t_{1}+\tau_{1}, \ldots, t_{q}+\tau_{q}\right)\right\rangle\right\rangle \\
& =\left\langle F\left(t_{1}, \ldots, t_{q}\right),\left\langle\delta^{\left(j_{1}, \ldots, j_{q}\right)}\left(\tau_{1}, \ldots, \tau_{q}\right), \phi\left(t_{1}+\tau_{1}+\tau_{1}^{0}, \ldots, t_{q}+\tau_{q}+\tau_{q}^{0}\right)\right\rangle\right\rangle \\
& =\left\langle F\left(t_{1}, \ldots, t_{q}\right),\left\langle\delta\left(\tau_{1}, \ldots, \tau_{q}\right),\left.(-1)^{|j|}\right|_{\phi} ^{\left(j_{1}, \ldots, j_{q}\right)}\left(t_{1}+\tau_{1}+\tau_{1}^{0}, \ldots, t_{q}+\tau_{q}+\tau_{q}^{0}\right)\right\rangle\right\rangle \\
& \left.=\left\langle F\left(t_{1}, \ldots, t_{q}\right),(-1)^{|j|}{ }_{\phi}^{\left(j_{1}, \ldots, j_{q}\right)}\left(t_{1}+\tau_{1}^{0}, \ldots, t_{q}+\tau_{q}^{0}\right)\right\rangle\right\rangle \\
& \left.=\left\langle F^{\left(j_{1}, \ldots, j_{q}\right)}\left(t_{1}-\tau_{1}^{0}, \ldots, t_{q}-\tau_{q}^{0}\right), \phi\right\rangle\right\rangle .
\end{aligned}
$$

Since this holds for all test functions, $\phi \in \mathscr{T}\left(\Re^{q}\right)$, we have

$$
F * \delta^{\left(j_{1}, \ldots, j_{q}\right)}\left(\tau_{1}-\tau_{1}^{0}, \ldots, \tau_{q}-\tau_{q}^{0}\right)=F^{\left(j_{1}, \ldots, j_{q}\right)}\left(t_{1}-\tau_{1}^{0}, \ldots, t_{q}-\tau_{q}^{0}\right) .
$$

We now consider a mother wavelet, $\psi(t) \in L^{2}\left(\Re^{q}\right)$ or $\mathscr{Y}\left(\Re^{q}\right)$ such as the one in expression (4.6). We consider a distribution, $F(t)$, where the support of $F(t)$ intersects the support of $\psi_{a}(t)$ and $\phi(t+\tau)$ in a bounded set, let us say $\Omega \subset \Re^{2 q}$. We then select a test function, $\lambda(t, \tau) \in \mathscr{T}\left(\Re^{2 q}\right)$ that is equal to one over some neighborhood of $\Omega$ and zero in a larger region as shown in Zemanian [39]. For such a distribution, $F(t)$, satisfying what we term the bounded intersection support property with the functions, $\psi_{a}(t)$ and $\phi(t+\tau)$, we then define the following wavelet transform for $F(t)$.

DEFINITION 5.5. The wavelet transform of a distribution, $F(t)$ satisfying the bounded intersection support property with $\psi_{a}(t)$ and $\phi(t+\tau)$ is given by

$$
\left(W_{\psi} F\right)(a) \triangleq F\left(t_{1}, \ldots, t_{q}\right) * \psi_{a}\left(\tau_{1}, \ldots, \tau_{q}\right)
$$

where

$$
\begin{aligned}
& \left\langle F\left(t_{1}, \ldots, t_{q}\right) * \psi_{a}\left(\tau_{1}, \ldots, \tau_{q}\right), \phi\right\rangle \\
& =\left\langle F\left(t_{1}, \ldots, t_{q}\right)\left\langle\psi_{a}\left(\tau_{1}, \ldots, \tau_{q}\right), \lambda\left(t_{1}, \ldots, t_{q}, \tau_{1}, \ldots, \tau_{q}\right) \phi\left(t_{1}+\tau_{1}, \ldots, t_{q}+\tau_{q}\right)\right\rangle\right\rangle
\end{aligned}
$$


As in Zemanian [39], this convolution product is well defined making our wavelet transform well defined.

We compute the wavelet transform of $\delta\left(t_{1}-b_{1}, \ldots, t_{q}-b_{q}\right)$ with a mother wavelet, $\psi\left(\tau_{1}, \ldots, \tau_{q}\right)$ which satisfies the bounded intersection support property with $\psi_{a}(\tau)$ and $\phi(t+\tau)$ whenever $\phi(t) \in \mathscr{D}\left(\Re^{q}\right)$. To this end we have

$$
\begin{aligned}
& \left\langle\delta(t-b) * \psi_{a}(t), \phi\right\rangle= \\
& \left\langle\delta(t-b), \int_{-\infty}^{\infty} \ldots \int_{-\infty}^{\infty} \frac{1}{\left.\sqrt{\prod_{i=1}^{q} a_{i}} \psi\left(\frac{\tau_{1}}{-a_{1}}, \ldots, \frac{\tau_{q}}{-a_{q}}\right) \lambda(t, \tau) \phi\left(t_{1}+\tau_{1}, \ldots, t_{q}+\tau_{q}\right) d t_{1} \ldots d t_{q}\right\rangle}\right. \\
& =\left\langle\delta(t-b), \sqrt{\prod_{i=1}^{q} a_{i}} \int_{-\infty}^{\infty} \cdots \int_{-\infty}^{\infty} \psi\left(s_{1}, \ldots, s_{q}\right) \lambda(t,-a s) \phi\left(t_{1}-a s_{1}, \ldots, t_{q}-a_{q} s_{q}\right) d s_{1} \ldots d s_{q}\right\rangle \\
& =\sqrt{\prod_{i=1}^{q} a_{i}} \int_{-\infty}^{\infty} \cdots \int_{-\infty}^{\infty} \psi\left(s_{1}, \ldots, s_{q}\right) \lambda(b,-a s) \phi\left(b_{1}-a_{1} s_{1}, \ldots, b_{q}-a_{q} s_{q}\right) d s_{1} \ldots d s_{q} \\
& =\int_{-\infty}^{\infty} \ldots \int_{-\infty}^{\infty} \psi\left(\frac{t_{1}-b_{1}}{-a_{1}}, \ldots, \frac{t_{q}-b_{q}}{-a_{q}}\right) \lambda(b, b-t) \phi\left(t_{1}, \ldots, t_{q}\right) d t_{1} \ldots d t_{q} \\
& =\left(\psi_{a}(t-b), \phi(t)\right)_{L^{2}\left(\Re^{q}\right)}
\end{aligned}
$$

Since this holds for all test functions, $\phi(t) \in \mathscr{D}\left(\Re^{q}\right)$, we have $\left(W_{\psi} \delta(t-b)\right)(a)=\psi_{a}(t-b)$. Clearly the "rough" delta functional is transformed to a "rather smooth" or "smooth" regular distribution, $\psi_{a}(t-b)$ depending on the smoothness of the mother wavelet $\psi(t)$ selected for the application.

We now extend our wavelet transform to a tempered distribution, $F \varepsilon \varphi^{\prime}\left(\Re^{q}\right)$ and a mother wavelet, $\psi(\tau) \varepsilon \varphi\left(\Re^{q}\right)$, following the convolution construction Zemanian [39].

DEFINITION 5.6. Given $F \varepsilon \varphi^{\prime}\left(\Re^{q}\right)$, a mother wavelet, $\psi(\tau) \varepsilon \varphi\left(\Re^{q}\right)$ and a test function $\phi \varepsilon \mathscr{T}\left(\Re^{q}\right)$. We then define the wavelet transform of $F$ to be

$$
\left(W_{\psi} F\right)(a) \triangleq F\left(t_{1}, \ldots, t_{q}\right) * \psi_{a}\left(\tau_{1}, \ldots, \tau_{q}\right)
$$

where

$$
\begin{aligned}
& \left\langle F\left(t_{1}, \ldots, t_{q}\right) * \psi_{a}\left(\tau_{1}, \ldots, \tau_{q}\right), \phi\left(t_{1}, \ldots, t_{q}\right)\right\rangle \triangleq \\
& \quad\left\langle F\left(t_{1}, \ldots, t_{q}\right) \times \phi\left(\tau_{1}, \ldots, \tau_{q}\right), \lambda\left(\tau_{1}, \ldots, \tau_{q}\right) \psi_{a}\left(\tau_{1}-t_{1}, \ldots, \tau_{q}-t_{q}\right)\right\rangle
\end{aligned}
$$

where $\lambda\left(\tau_{1}, \ldots, \tau_{q}\right) \varepsilon \mathscr{T}\left(\Re^{q}\right)$ and equals one over a neighborhood of the support of $\phi\left(t_{1}, \ldots, t_{q}\right) \in \mathscr{T}\left(\Re^{q}\right)$ and zero in a larger region. 


\section{WAVELET TRANSFORMS OF GENERALIZED FOCK SPACES}

The technique of extending the wavelet transform to our generalized Fock spaces is in the spirit of extending the Fourier and Hankel transforms to generalized Fock spaces as conducted by Schmeelk [4.5] respectively.

For this section we consider the functions, $\mathcal{H}_{b}=\left(M_{p}(\cdot)\right)_{p \varepsilon N_{0}}$, to be

$$
M_{p}\left(t_{1}, \ldots, t_{q}\right)=\left[\left(1+t_{1}^{2}\right) \ldots\left(1+t_{q}^{2}\right)\right]^{p}
$$

We now define the wavelet transform on functional, $\Phi$, having the representative,

$$
\Phi \Leftrightarrow\left[\begin{array}{c}
\phi_{0} \\
\phi_{1}\left(t_{1}\right) \\
\vdots \\
\phi_{q}\left(t_{1}, \ldots, t_{q}\right) \\
\vdots
\end{array}\right],
$$

and satisfying the norm conditions,

$$
\|\mid \Phi\|_{s, \mu, m}^{(p)}=\sup _{q \varepsilon N_{0}} \frac{\left\|\phi_{q}\right\|_{p} \cdot m_{q}}{s^{q}}<\infty,
$$

for all $p \varepsilon N_{0}$.

DEFINITION 6.3 Given a normalized mother wavelet $\psi(t) \varepsilon L^{2}\left(\Re^{q}\right), q \geq 1$, the wavelet transform of $\Phi$ denoted $W_{\Phi}(a, \tau)$ is

$$
\left.\begin{array}{c}
W_{\Phi}(a, \tau):\left[\begin{array}{c}
\phi_{0} \\
\phi_{1}\left(t_{1}\right) \\
\vdots \\
\phi_{q}\left(t_{1}, \ldots, t_{q}\right) \\
\vdots
\end{array}\right] \mapsto \\
{\left[\phi_{1}\left(t_{1}\right) * \psi_{a_{1}}\left(t_{1}\right)\right]\left(\tau_{1}\right)} \\
\vdots \\
{\left[\phi_{q}\left(t_{1}, \ldots, t_{q}\right) * \psi_{a_{1}, \ldots, a_{q}}\left(t_{1}, \ldots, t_{q}\right)\right]\left(\tau_{1}, \ldots, \tau_{q}\right)} \\
\vdots
\end{array}\right] .
$$

LEMMA 6.5. The wavelet transform of $\Phi, W_{\Phi}(a, \tau)$, is well defined for every $\Phi \varepsilon \Gamma^{\mu, \mathcal{H}, s}$, having the representation given in expression (6.1) together with a normalized mother wavelet. 
PROOF: Let $\Phi \varepsilon \Gamma^{s, \mu, \mathcal{A}}$ for some $s>1$. We consider

$$
\begin{aligned}
& \left|\phi_{0}+\sum_{q=1}^{\infty}\left[\phi_{q}\left(t_{1}, \ldots, t_{q}\right) * \psi_{a}\left(t_{1}, \ldots, t_{q}\right)\right]\left(\tau_{1}, \ldots, \tau_{q}\right)\right| \\
& \leq\left|\phi_{0}\right|+\left|\sum_{q=1}^{\infty} \frac{1}{\sqrt{\prod_{i=1}^{q} a_{i}}} \int_{-\infty}^{\infty} \cdots \int_{-\infty}^{\infty} \phi_{q}\left(t_{1}, \ldots, t_{q}\right) \psi\left(\frac{\tau_{1}-t_{1}}{-a_{1}} \ldots \frac{\tau_{q}-t_{q}}{-a_{q}}\right) d t_{1}, \ldots d t_{q}\right| \\
& =\left|\phi_{0}\right|+\left|\sum_{q=1}^{\infty} \int_{-\infty}^{\infty} \ldots \int_{-\infty}^{\infty} \phi_{q}\left(t_{1}, \ldots, t_{q}\right) \psi^{\left(a_{1}, \ldots, a_{q} ; \tau_{1}, \ldots, \tau_{q}\right)}\left(t_{1}, \ldots, t_{q}\right) d t_{1}, \ldots d t_{q}\right| \\
& \leq\left|\phi_{0}\right|+\sum_{q=1}^{\infty}\left[\int_{-\infty}^{\infty} \cdots \int_{-\infty}^{\infty}\left|\phi_{q}\left(t_{1}, \ldots, t_{q}\right)\right|^{2} d t_{1}, \ldots d t_{q}\right] \text {. } \\
& {\left[\int_{-\infty}^{\infty} \ldots \int_{-\infty}^{\infty}\left|\psi^{\left(a_{1}, \ldots, a_{q} ; \tau_{1}, \ldots, \tau_{q}\right)}\left(t_{1}, \ldots, t_{q}\right)\right|^{2} d t_{1}, \ldots d t_{q}\right]} \\
& =\left|\phi_{0}\right|+\sum_{q=1}^{\infty}\left[\int_{-\infty}^{\infty} \cdots \int_{-\infty}^{\infty}\left|\phi_{q}\left(t_{1}, \ldots, t_{q}\right)\right|^{2} d t_{1}, \ldots d t_{q}\right] \\
& =\left|\phi_{0}\right|+\sum_{q=1}^{\infty}\left[\int_{-\infty}^{\infty} \cdots \int_{-\infty}^{\infty} \frac{M_{q}^{2}\left(t_{1}, \ldots, t_{q}\right)\left|\phi_{q}\left(t_{1}, \ldots, t_{q}\right)\right|^{2}}{M_{q}^{2}\left(t_{1}, \ldots, t_{q}\right)} d t_{1}, \ldots d t_{q}\right] \\
& \leq\left|\phi_{0}\right|+\sum_{q=1}^{\infty}\left\|\phi_{q}\right\|_{p}^{2} \cdot \pi^{q} \frac{m_{q}^{2}}{s^{2 q}} \frac{s^{2 q}}{m_{q}^{2}} \\
& =\left|\phi_{0}\right|+\sum_{q=1}^{\infty}\left(\frac{\left\|\phi_{q}\right\|_{p} \cdot m_{q}}{s^{q}}\right)^{2} \cdot \frac{\pi^{q} s^{2 q}}{m_{q}^{2}} \\
& \leq\left|\phi_{0}\right|+C_{p}^{2} \sum_{q=1}^{\infty} \frac{\pi^{q} s^{2 q}}{m_{q}^{2}}<\left|\phi_{0}\right|+C_{p}^{2} \sum_{q=1}^{\infty} \frac{s_{1}^{q}}{m_{q}}<\infty
\end{aligned}
$$

where $s_{1}=\pi s^{2}$. In view of Lemma 3.1 found in Schmeelk and Takaći [6], page 268, the last sum is finite.

We now consider the space, $\left(\Gamma^{\mu, M}\right)^{\prime}$ defined in expression (3.10) and recall that an $F \varepsilon$ $\left(\Gamma^{\mu, M}\right)^{\prime}$ has representation,

$$
F \Leftrightarrow\left[F_{0}, \ldots, F_{q}, \ldots\right]
$$

where $F_{0} \varepsilon \mathbb{C}$ and $F_{q} \varepsilon 96^{\prime}(\mathcal{M}), q \geq 1$ and all have order $\leq p$. Moreover the $F$ then satisfies the norm condition

$$
\sum_{q=0}^{\infty}\left\|F_{q}\right\|_{-p} \frac{s^{q}}{m_{q}}<\infty
$$

As an example of such a generalized Fock functional, we consider 


$$
\delta(t-b) \Leftrightarrow\left[\begin{array}{c}
1 \\
\delta\left(t_{1}-b_{1}\right) \\
\vdots \\
\delta\left(t_{1}-b_{1}\right) \times \ldots \times \delta\left(t_{q}-b_{q}\right) \\
\vdots
\end{array}\right]
$$

where it is clear that $1+\sum_{q=1}^{\infty}\left\|\delta\left(t_{1}-b_{1}\right) \times \ldots \times \delta\left(t_{q}-b_{q}\right)\right\|_{-p} \frac{s^{q}}{m_{q}}<\infty$ for every $p$. We then define the wavelet transform of our generalized Fock functional to be

$$
\left(W_{\psi} F\right)(a) \Leftrightarrow\left[\begin{array}{c}
F_{0} \\
W_{\psi}\left(F_{1}\right)\left(a_{1}\right) \\
\vdots \\
W_{\psi}\left(F_{q}\right)\left(a_{1}, \ldots, a_{q}\right) \\
\vdots
\end{array}\right]
$$

We compute the wavelet transform of the generalized Fock functional given in expression (6.6) with a normalized mother wavelet and obtain

$$
\left[\begin{array}{c}
F_{0} \\
\psi_{a_{1}}\left(t_{1}-b_{1}\right) \\
\vdots \\
\psi_{a_{1}, a_{2}, \ldots, a_{q}}\left(t_{1}-b_{1}, \ldots, t_{q}-b_{q}\right) \\
\vdots
\end{array}\right]
$$

We observe that each entry in expression (6.3) for $q \geq 1$ satisfies the following norm condition.

$$
\begin{aligned}
& \left\|\psi_{a_{1}, a_{2}, \ldots, a_{q}}\left(t_{1}-b_{1}, \ldots, t_{q}-b_{q}\right)\right\|_{-p} \\
& =\sup _{\|\phi\|_{p} \leq 1}\left\{\left|\int_{-\infty}^{\infty} \cdots \int_{-\infty}^{\infty} \psi\left(\frac{t_{1}-b_{1}}{-a_{1}}, \ldots, \frac{t_{q}-b_{q}}{-a_{q}}\right) \phi\left(t_{1}, \ldots, t_{q}\right) d t_{1} \ldots d t_{q}\right|\right\} \\
& \quad \leq \sup _{\|\phi\|_{p} \leq 1}\left\{\left|\int_{-\infty}^{\infty} \cdots \int_{-\infty}^{\infty} \psi\left(\frac{t_{1}-b_{1}}{-a_{1}}, \ldots, \frac{t_{q}-b_{q}}{-a_{q}}\right)\right|^{2} d t_{1} \ldots d t_{q}\right\}^{\frac{1}{2}}
\end{aligned}
$$




$$
\left\{\int_{-\infty}^{\infty} \ldots \int_{-\infty}^{\infty}\left|\phi\left(t_{1}, \ldots, t_{q}\right)\right|^{2} d t_{1} \ldots d t_{q}\right\}^{\frac{1}{2}} \leq \pi^{q}
$$

Thus we immediately have that our wavelet transform of our generalized Fock functional given in example (6.8) satisfies the norm condition,

$$
1+\sum_{q=1}^{\infty}\left\|\psi_{a_{1}, a_{2}, \ldots, a_{q}}\left(t_{1}-b_{1}, \ldots, t_{q}-b_{q}\right)\right\|_{-p} \frac{s^{q}}{m_{q}} \leq 1+\sum_{q=1}^{\infty} \frac{\pi^{q} s^{q}}{m_{q}}<\infty
$$

We conclude with considering the general case given in expression (6.7). We require that our generalized Fock functional has each entry, $F_{q}, q \geq 1$, and normalized mother wavelet satisfying the bounded support intersection property. For this situation we then have

$$
\begin{gathered}
\left\|\mid W_{\psi}(F)(a)\right\|\left\|_{s, \mu, m}^{(-p)}=\sum_{q=0}^{\infty}\right\| W_{\psi}\left(F_{q}\right)\left(a_{1}, \ldots, a_{q}\right) \|_{-p} \frac{s^{q}}{m_{q}} \\
=\left|F_{0}\right|^{2}+\sum_{q=1}^{\infty}\left\|W_{\psi}\left(F_{q}\right)\left(a_{1}, \ldots, a_{q}\right)\right\|_{-p} \frac{s^{q}}{m_{q}} .
\end{gathered}
$$

Now for each $q \geq 1$ in expression (6.11) we have

$$
\begin{aligned}
& \left\|W_{\psi}\left(F_{q}\right)\left(a_{1}, \ldots, a_{q}\right)\right\|_{-p} \\
& =\left\{\left\langle F\left(t_{1}, \ldots, t_{q}\right) \times \psi_{a}\left(\tau_{1}, \ldots, \tau_{q}\right), \lambda\left(t_{1}, \ldots, t_{q}, \tau_{1}, \ldots, \tau_{q}\right) \phi\left(t_{1}+\tau_{1}, \ldots, t_{q}+\tau_{q}\right)\right\rangle \mid:\left\|\phi_{p}\right\| \leq 1\right\} \\
& =\|\left\langle F\left(t_{1}, \ldots, t_{q}\right), \int_{-\infty}^{\infty} \ldots \int_{-\infty}^{\infty} \psi\left(\frac{\tau_{1}}{-a_{1}}, \ldots, \frac{\tau_{q}}{-a_{q}}\right) \lambda\left(t_{1}, \ldots, t_{q}, \tau_{1}, \ldots, \tau_{q}\right) \phi\left(t_{1}+\tau_{1}, \ldots, t_{q}+\tau_{q}\right)\|:\| \phi_{p} \| \leq 1\right. \\
& =\left\{\mid\left\langle F\left(t_{1}, \ldots, t_{q}\right) \int_{-\infty}^{\infty} \ldots \int_{-\infty}^{\infty} \psi\left(\frac{T_{1}-t_{1}}{-a_{1}}, \ldots, \frac{T_{q}-t_{q}}{-a_{q}}\right) \lambda\left(t_{1}, \ldots, t_{q}, T_{1}-t_{1}, \ldots, T_{q}-t_{q}\right)\right.\right. \\
& \left.\left.\phi\left(T_{1}, \ldots, T_{q}\right) d T_{1}, \ldots, d T_{q}\right) \mid:\left\|\phi_{p}\right\| \leq 1\right\} \\
& \left\|F\left(t_{1}, \ldots, t_{q}\right)\right\|_{s, \mu, m}^{(-p)} \| \int_{-\infty}^{\infty} \ldots \int_{-\infty}^{\infty} \psi\left(\frac{T_{1}-t_{1}}{-a_{1}}, \ldots, \frac{T_{q}-t_{q}}{-a_{q}}\right) \lambda\left(t_{1}, \ldots, t_{q}, T_{1}-t_{1}, \ldots\right. \\
& \left.T_{q}-t_{q}\right) \phi\left(T_{1}, \ldots, T_{q}\right) d T_{1}, \ldots, d T_{q} \|_{s, \mu, m}^{(p)} \text {. }
\end{aligned}
$$

for all $\|\phi\|_{p} \leq 1$. Since our mother wavelet is normalized and our test functions, $\phi$, satisfy $\|\phi\|_{p} \leq 1$ we immediately have our sum in expression (6.11) to be finite. 


\section{REFERENCES}

[1] SCHMEELK, JOHN, "Infinite Dimensional Parametric Distributions," Applicable Analysis an Intemational Journal, 24, No.4, (1987), 291-319.

[2] SCHMEELK, JOHN, "Infinite Dimensional Fock Spaces and Associated Creation and Annihilation Operators," J. of Analysis and Applications, 135, No.2, (1988), 432-461.

[3] SCHMEELK, JOHN, Multiplication and Mixed Differentiation in Generalized Fock Spaces, Journal of Analysis and Applications, 165, No.1, (1992), 216-238.

[4] SCHMEELK, JOHN, Fourier Transforms in Generalized Fock Spaces, International Journal of Mathematics and Mathematical Science, 13, No.3, (1990), 431-442.

[5] SCHMEELK, JOHN, "Hankel Transforms in Generalized Fock Spaces," Intemational J. of Mathematics and Mathematical Science, 17, No.2, (1994), 259-271.

[6] SCHMEELK, JOHN and TAKAČI, ARPAD, "Ultra Annihilation and Creation Operators," Portugaliae Mathematica, 49, No.3, (1992), 263-279.

[7] SCHMEELK, JOHN and TAKAČI, ARPAD, "Fock Spaces and Regularly Varying Functions," Bulletin of Applied Mathematics and Mechanics, Budapest, Hungary, to appear.

[8] SCHMEELK, JOHN and TAKAČI, ARPAD, "Quasiasymptotic Behavior in a Generalized Fock Space," Review of Research. Faculty of Science, Institute of Mathematics, University of Novi Sad, Yugoslavia, in print.

[9] MIKUSINSKI, PIOTR, and MOTT, MICHAEL, L., "The Integral Wavelet Transform of Convolutors," (1995), Preprint.

[10] MIKUSINSKI, PIOTR, and TAYLOR, MICHAEL. D., "Toward a Unified Theory of Generalized Functions: Convergence," Math. Nachr. 161, (1993), 27-43.

[11] STRANGE, GILBERT, "Wavelet Transforms versus Fourier Transforms," Bulletin of the AMS, 28, No.2, (1993), 288-305.

[12] VON BAEYER, HANS, CHRISTIAN, "Wave of the Future," Discover, 16, No.5, (1995), 6874.

[13] GROSSMANN, A., and MORLET, J., "Decomposition of Hardy 'Functions into Square Integrable Wavelets of Constant Shape," Siam J. Math. Anal., 15, No.4, (1984), 723-736.

[14] WALTER, GILBERT, G., “Approximation of the Delta Function by Wavelets," J. of Approximation Theory, 71, No.3, (1992), 329-343.

[15] WALTER, GILBERT, G., "Sampling Bandlimited Functions of Polynomial Growth," SLAM J. Math. Anal., 19, No.5, (1988), 1198-1203.

[16] WALTER, GILBERT, G., "Negative Spline Wavelets," J. Math. Anal. \& Applications, 177, No.1, (1993), 239-253.

[17] WALTER, GILBERT, G., "Wavelet Subspaces with an Oversampling Property," Indag. Mathem., N.S. 4, No.4, (1993), 499-507.

[18] WALTER, GILBERT, G., "Pointwise Convergence of Wavelet Expansions," $J$. of Approximation Theory, 80, No.1, (1995), 108-118. 
[19] WALTER, GILBERT, G., "Translation and Dilation Invariance in Orthogonal Wavelets," Applied and Computational Harmonic Analysis, 1, (1994), 344-349.

[20] WALTER, GILBERT, G., "Wavelets and Other Orthogonal Systems with Applications," CRC Press Inc., FL, 1994.

[21] MALLAT, STEPHANE, G., "Multifrequency Channel Decompositions of Images and Wavelet Models," IEEE Transactions on A coustics, Speech, and Signal Processing, 37, No.12, (1989), 2091-2210.

[22] MALLAT, STEPHANE, G., “A Theory for Multiresolution Signal Decomposition: The Wavelet Representation," IEEE Transactions on Pattem Analysis and Machine Intelligence, 11, No.7, (1989), 674-693.

[23] MALlAT, STEPHANE, G., "Zero-Crossings of a Wavelet Transform," IEEE Trans. on Information Theory, 37, No.4, (1991), 1019-1033.

[24] MALLAT, STEPHANE, G., "Multiresolution Approximations and Wavelet Orthonormal Bases of $\mathbf{L}^{2}(\mathrm{R}), "$ Trans. Amer. Math. Soc., 315, No.1, (1989), 69-87.

[25] MEYER, YVES, “Ondelettes et Algorithmes Concurrents," Hermann, Paris, 1992.

[26] DAUBECHIES, INGRID, "Ten Lectures on Wavelets," Society for Industrial and Applied Mathematics, PA, 1992.

[27] LIM, JAE, S., "Two-Dimensional Signal and Image Processing," Prentice-Hall, Englewood Cliffs, NJ, 1990.

[28] KOMATSU, H., "Ultradistributions, I, Structural Theorems and a Characterization," $J$. of the Faculty of Science, Tokyo, Section 1A Mathematics, 20, (1973), 25-105.

[29] GELFAND, I., M., and SHILOV, G.,E., "Generalized Functions, Vol.II," Academic Press, NY, 1986.

[30] PILIPOVIC, S., and TAKAČI, A., "Space H'(M $\left.\mathrm{M}_{\mathrm{p}}\right)$ and Convolutors," Proc. of the Moscow Conference on Generalized Functions, Moscow, (1981), 415-427.

[31] AKANSU, ALI, N., and HADDAD, RICHARD, A., "Multiresolution Signal Decomposition," Academic Press, MA, 1992.

[32] BENEDETTO, JOHN, J., and FRAZIER, MICHAEL, W. Editors, "Wavelets Mathematics and Applications," CRC Press, FL, 1994.

[33] BEYLKIN, G., COIFMAN, R., and ROKHLIN, V., "Fast Wavelet Transforms and Numerical Algorithms I," Communications on Pure and Applied Mathematics, 44, (1991), 141-183.

[34] HOLSCHNEIDER, M., "Wavelets An Analysis Tool," Oxford Science Publications, Oxford, 1995.

[35] KAISER, GERALD, “A Friendly Guide to Wavelets,” Birkhauser, Boston, 1994.

[36] KOORNWINDER, TOM, H., Editor, "Wavelets: An Elementary Treatment of Theory and Applications," World Scientific, New Jersey, 1993.

[37] RUSKAI, MARY, BETH, editor, et al, "Wavelets and their Applications," Jones and Bartlett Publishers, MA, 1992. 
[38] SCHUMAKER, LARRY, L. and WEBB, GLENN, Editors, "Recent Advances in Wavelet Analysis," Academic Press, MA, 1994.

[39] ZEMANIAN, A.,H., "Distribution Theory and Transform Analysis," McGraw-Hill, NY, 1965.

[40] SAITOH, SABUROU, "Analyticity in the Meyer Wavelets," Preprint.

[41] VLADIMIROV, V.,S., "Equations of Mathematical Physics," Marcel Dekker Inc., NY, 1971.

[42] CHUI, CHARLES, K., “An Introduction to Wavelets," Academic Press, MA, 1992.

[43] CHUI, CHARLES, K., Editor, "Wavelets: A Tutorial in Theory and Applications," Academic Press, MA, 1992.

[44] CODY, MAC A., "The Wavelet Packet Transform," Dr. Dobb's Journal, (April-1994), 44-52.

[45] COMBES, J.,M., GROSSMANN, and A., TCHAMITCHIAN, Ph., Editors, "Wavelets TimeFrequency Methods and Phase Space," Proc. of the International Conference, Marseille, France, 1987, Springer-Verlag, NY, 1989.

[46] DAUBECHIES, INGRID, "Time-Frequency Localization Operators: A Geometric Phase Space Approach," IEEE Trans. on Inf. Theory, 34, No.4, (1988), 605-612.

[47] DAUBECHIES, INGRID, The Wavelet Transform, Time-Frequency Localization and Signal Analysis, IEEE Transactions on Information Theory, 36, No. 5, (1990), 961-1005.

[48] GOODMAN, T. N. T., and LEE, S. L., TANG, W. S., "Wavelets in Wandering Subspaces," Trans. A mer. Math. Soc., 338, No.2, (1993), 639-654.

[49] HEIL, CHRISTOPHER, E., and WALNUT, DAVID, F., "Continuous and Discrete Wavelet Transforms," SLAM Review, 31, No.4, (1989), 626-666.

[50] KAISER, GERALD, An Algebraic Theory of Wavelets. I. Operational Calculus and Complex Structure, SIAM J. Math. Anal., 23, No.1, (1992), 222-243.

[51] KELlY, SUSAN, and KON, MARK, A., and RAPHAEL, LOUISE, A., "Pointwise Convergence of Wavelet Expansions," Bulletin of the AMS, 30, No.1, (1994), 87-94.

[52] KELLY, SUSAN, and KON, MARK, A.,and RAPHAEL, LOUISE,A., "Local Convergence for Wavelet Expansions," ICM-94, Zurich, Switzerland, Aug.3-11, 1994, Preprint.

[53] LAI, MING-JUN, “A Matrix Approach to Computations of Various Wavelets," Department of Mathematics, University of Georgia, Athens, Ga. 30602 Preprint, (1993).

[54] LIVERMAN, T., P., G., “Generalized Functions and Direct Operational Methods," PrenticeHall, Englewood Cliffs, NJ, 1964.

[55] STRANGE, GILBERT, "Wavelets and Dilation Equations: A Brief Introduction," SLAM Review, 31, No.4, (1989), 614-627.

[56] VETTERLI, MARTIN, and HERLEY, CORMAC, "Wavelets and Filter Banks: Theory and Design," IEEE Transactions on Signal Processing, 40, No.9, (1992), 2207-2232. 


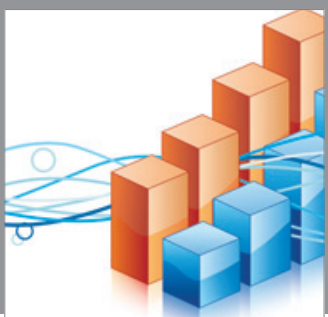

Advances in

Operations Research

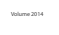

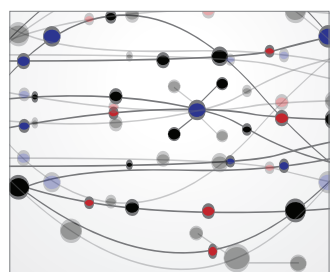

\section{The Scientific} World Journal
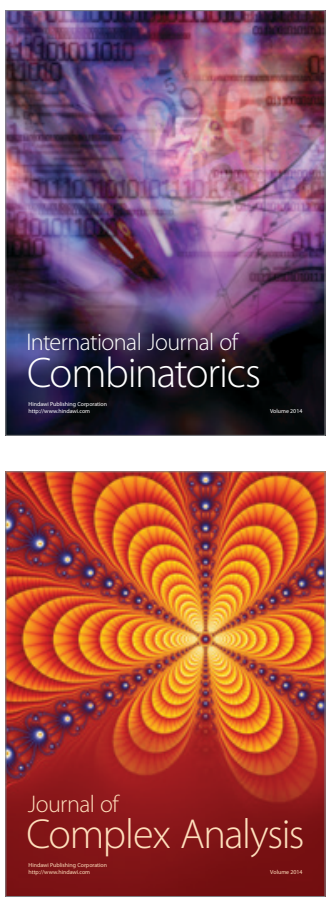

International Journal of

Mathematics and

Mathematical

Sciences
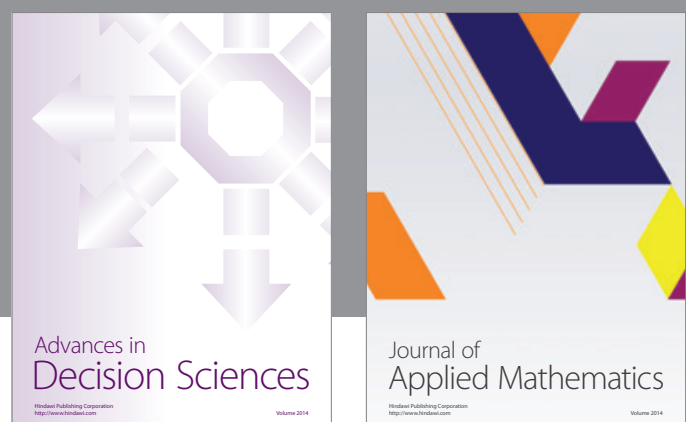

Journal of

Applied Mathematics
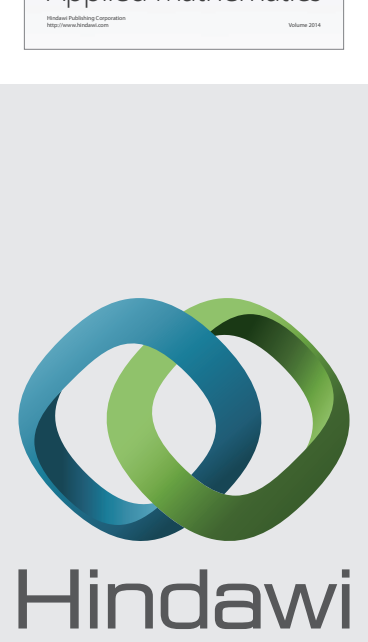

Submit your manuscripts at http://www.hindawi.com
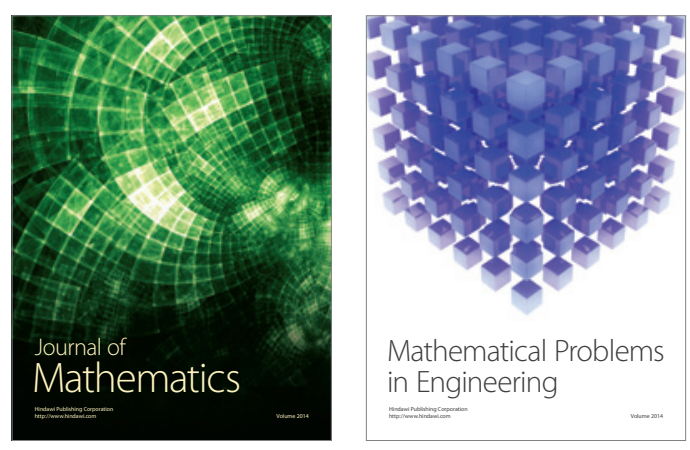

Mathematical Problems in Engineering
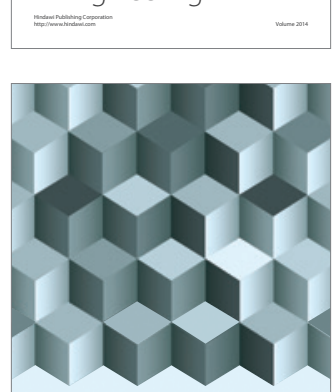

Journal of

Function Spaces
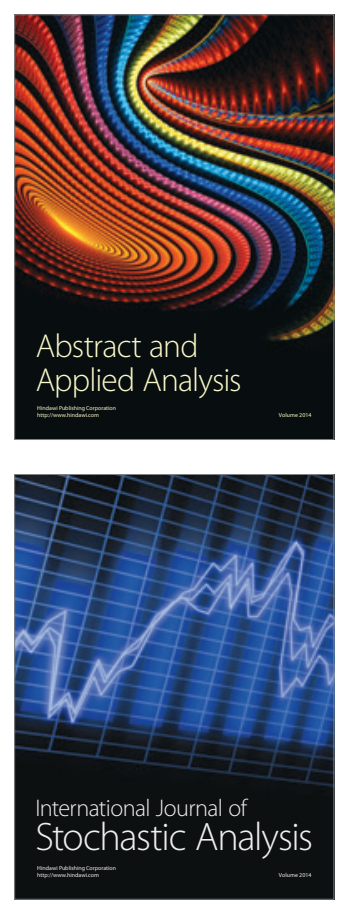

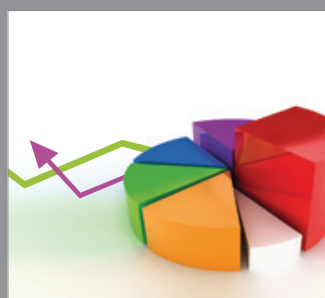

ournal of

Probability and Statistics

Promensencen
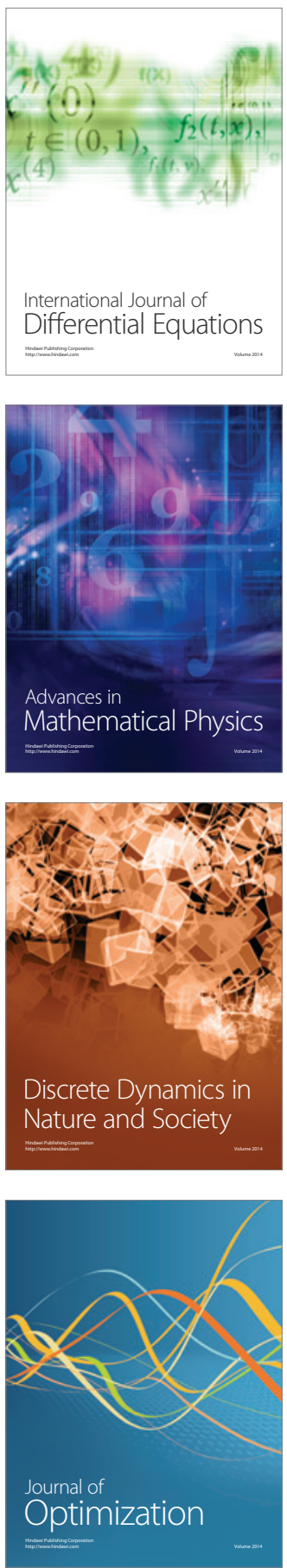